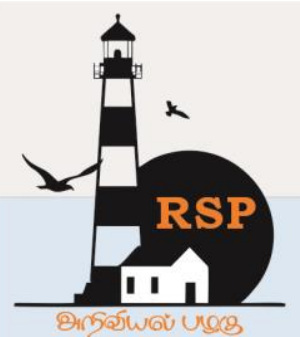

INTERNATIONAL RESEARCH JOURNAL ON

e-ISSN : 2582 - 4376 ADVANCED SCIENCE HUB Open Access

RSP SCIENCE HUB

(The Hub of Research Ideas)

Available online at www.rspsciencehub.com

\title{
Implementation Of AI Based Relaying Scheme For Overcurrent Protection Using Numerical Relay Development Environment Board
}

Shagila $s^{1}$,Dr.R.Rajeswari ${ }^{2}$

${ }^{1}$ P.G.Scholar ,Dept of EEE., Govt. College of technology, Coimbatore, India

${ }^{2}$ ASSOC PROF,Dept of EEE.,Govt.College of technology, Coimbatore, India shagilaselvam13196@gmail.com ${ }^{1}$

\begin{abstract}
Numerical overcurrent Relay is a type of protective relay that operates when a pre-set value exceeds a charge current. Because of its standard technology, numerical relay is better known as present generation relay. Overcurrent relay protects power systems in any aspect of the power network, i.e. transmission lines, transformers, generators or motors, against excessive currents. A relay program that is over-current is coded using the MPLAB platform. With the help of MATLAB Simulation the overcurrent is achieved. By varying the source voltage and the load resistance, we can find the usual load current values (In rms) and automatically increase the load circuit value when the short circuit element is inserted in that model that is being viewed as being overcurrent. Overcurrent data is trained in Artificial Intelligence (Neural Network) and implemented through coding in the NRDE Board. In NRDE testing software, a trip command is observed when overcurrent condition is reached.
\end{abstract}

Keywords: power system protection, Numerical relay, Overcurrent relay, neural network.

\section{Introduction}

The paper explains the overcurrent relay output in power systems, with the aid of ANNs. The distributed computing systems inspired by the biological nervous system[1-4] are artificial neural networks (ANNs). Artificial neural network consists of simple highly interconnected processing units called neurons each performing two functions aggregating its inputs from the external environment and producing an output from the aggregated inputs. A relation between a pair of neurons has a numerical strength attached to it called synaptic weight. ANN 's growth includes two phases: the process of preparation or learning, and the stage of testing. ANN training is done by presenting examples named training set to the network. During training, the synaptic weights are modified to model the problem. Once the network has learned the problem, new unknown patterns can be tested and its efficiency can be checked. (Phase testing). ANN can be categorized as supervised ANN or unsupervised ANN, depending on the training Imparted.

Numerical overcurrent relay is a form of protective relay that operates when a preset value is exceeded by the charge current. In an electrical power system, overcurrent or excess current is a situation where there is a greater than expected electrical current through a conductor, resulting in excessive heat generation, and the possibility of fire or equipment damage. Possible causes for overcurrent are short circuit, excessive load, incorrect design or a ground fault.[5-7] 
Numeric relays should meet the basic protective requirements. It will in real time acquire, sample, and convert the analog current signals from a power system into digital form. The designed relay was extensively tested for different load current values and two different tripping features. Overcurrent relays are the most inexpensive security relays used in delivery networks as the main / backup protection and as the backup protection in transmission and subtransmission networks.[8-10]

\section{Numerical relay}

Numeric protection relays protect transformers of power and distribution systems from faults of various types. For power transformers, such faults include distance safety, line differential, pilot wire, low impedance busbar, high impedance differential, frequency, voltage, circuit breaker failure, auto reclosing, and faults in synchronicity. These failures include protection against overcurrent, under or overvoltage, directional overcurrent, and feeder manager relay failures for power distribution systems.

Numeric safety relays are digital systems in constant contact through menu-driven interfaces with substation automation systems. We have binary inputs, outputs, and programmable logic configurables. They track, calculate, and report electrical values, faults, delays, and events. Numerical security relays feature high-speed operation and multi-functionality, offering selectivity and reliability improvements. As they detect faults with automatic supervision, they make power systems highly reliable while at the same time being compact in size and consuming very low power. Numerical safety relays are automated systems that communicate via menudriven interfaces in constant contact with substation automation systems.

They have binary inputs, outputs, and programmable logic configurability. They track, calculate, and record electrical values, faults, disruptions, and happenings. Numerical safety relays feature high-speed operation and multifunctionality, offering improved selectivity and stability. Since they detect faults with automatic control, they make power systems highly efficient, while being compact in size and consuming very low power.

\subsection{Overcurrent relay}

An Over-Current Relay is a form of protective relay that protects against overcurrent; it works when the current of load exceeds a preset value. This relay uses the current transformer (CT) and is calibrated by comparing the measured values with preset values to operate at or above a specific current level. If the current value of the input exceeds the value of the pickup, the relay detects an overcurrent and gives the circuit breaker a signal which opens its contact to disconnect the protected equipment. The relay sends a signal instantly after the fault has been picked up (in the case of instant overcurrent relays) or it can wait for a specific time before issuing a trip signal (in the case of overcurrent relays). This time delay is also known as the relay 's operating time, and is calculated by the relay on the basis of the microprocessor 's protective algorithm. Typically numerical OCRs allow the user to define custom time-current characteristics either graphically or in table form. Numeric security relays are equipped with multiple microprocessors. Inside the relay each microprocessor performs software functions such as executing protective algorithms and scheme logic, processing sensor signals, controlling output relays, and handling the human interface.

Typically numerical OCRs allow the user to define custom time-current characteristics either graphically or in table form. Numeric security relays are equipped with multiple microprocessors. Inside the relay each microprocessor performs software functions such as executing protective algorithms and scheme logic, processing sensor signals, controlling output relays, and handling the human interface.

\section{Artificial Neural Network}

In deep learning a computer model learns directly from pictures, text or sound to perform classification tasks. Deep learning models can achieve state-of-the-art accuracy, sometimes surpassing performance on a human level. Models are trained using a large set of labeled data and architectures of the neural network, which contain many layers.

Supervised ANN For its training, supervised ANN requires the input sets and outputs. The output of the ANN is compared with the desired output (target) during the training, and 
the difference (error) is minimized by using some algorithm. This training is repeated until an acceptable level is achieved for the actual output. Supervised ANN may be a feed forward or nonrecurring network such as Multi Layer Perceptron, Functional Link Net, and Radial Basis Function, or a feedback or recurring ANN such as Hopfield, often used in power system applications.

Numerous forms of neural networks are available, or may be in the development stage. They can be categorized according to their structure, data flow, use of neurons and their density, layers and activation filters in depth, etc.

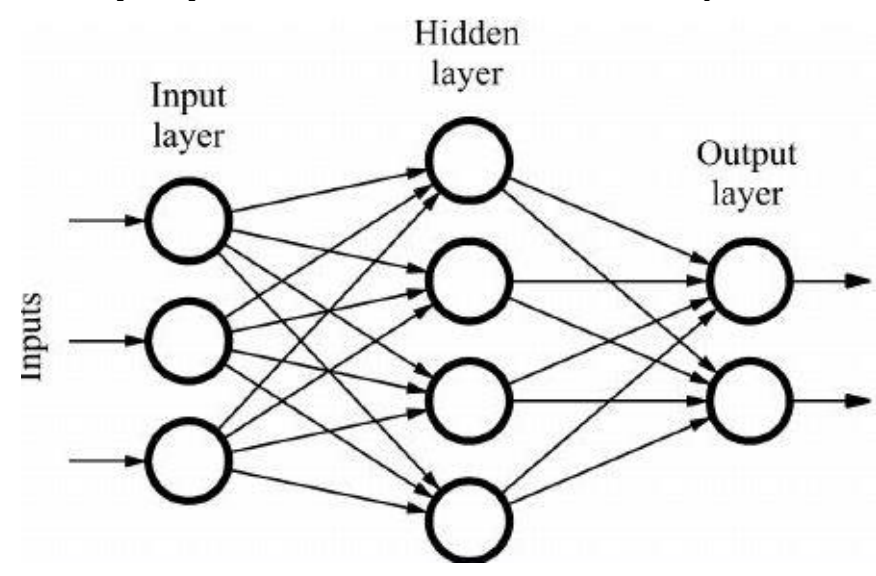

Fig.1. Neural Network model

Input layer represents the input vector dimensions. Hidden layer represents the intermediate nodes dividing the input space into regions with (soft) boundaries. It takes in a weighted input set and outputs output through an activation function. The output layer represents the neural network output.

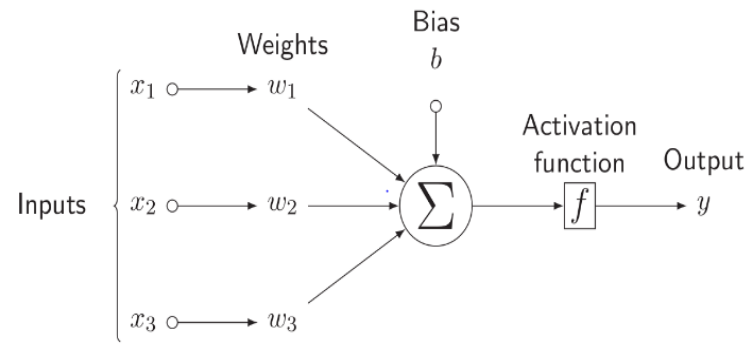

Fig.2. Example of Feedforward Network

Weights are numerical values, multiplied by inputs. They 're updated to reduce the loss in back propagation. Weights are, in plain words, machine-learned values from Neural Networks.
Depending on the difference between expected outputs and training inputs, they self-adjust. Activation Function: is a mathematical formula that makes the neuron turn ON / OFF.

\subsection{Feed Forward Neural Networks}

Simplest form of neural networks where input data travels in only one direction, passes through artificial neural nodes and exits through output nodes. Where there may or may not be hidden layers there are layers of input and output. On this basis, neural networks can be further classified as single layered or multilayered feed forward. The number of layers depends upon the function's complexity. It has uni-directional propagation of the forward but no propagation backwards. Weights here are static. Activation function is driven by weight-multiplied inputs. To do so, the activation classification function or activation step function is used. For example: if it is above threshold the neuron is activated and the neuron produces 1 as output. The neuron is not activated if it is considered as -1 below the threshold. They are relatively easy to maintain, and equipped to handle data that contains a lot of noise.

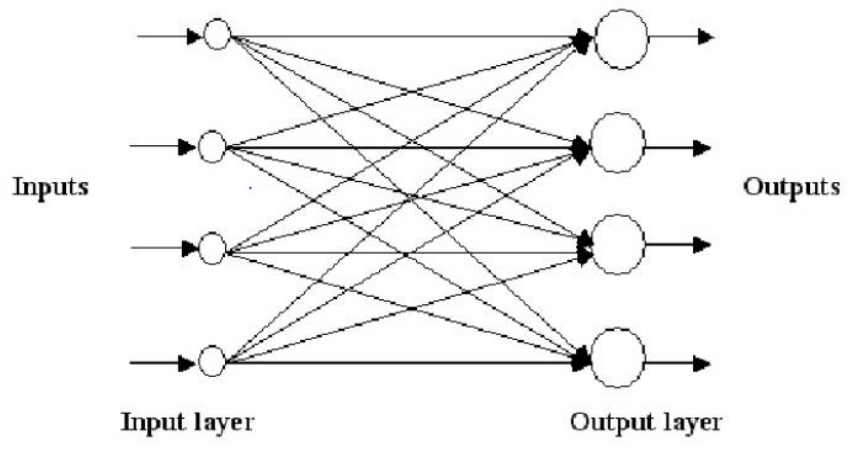

Fig.3. Feed forward model

A multilayer perceptron ( MLP) is a deep, neural artificial network. A neural network is composed of node layers which, depending on the nodes of the previous layer, activate at different levels. When thinking about neural networks, isolating your thinking to a single node in the network may be helpful. Multilayer perceptron refers to a neural network with at least three node 


\section{www.rspsciencehub.com}

layers, an input layer, a number of intermediate layers and an output layer. For adjacent layers, every node in a given layer is connected to each node. It's just that input layer, it's the way the network takes in data. The intermediate layers are the network's computational machine, they actually transform the input into the output. Output layer is the way neural network results are obtained. In a simple network where the answers are binary, there would probably only be one node in the output layer, which produces a likelihood of logistical regression.

\subsection{Learning methods}

Unsupervised learning is a type of machine learning that searches for previously undetected patterns in a data set with no pre-existing labels and a minimum of human oversight. Unlike supervised learning, which usually uses humanlabeled data, unsupervised learning, also known as self-organization, allows modeling of densities of probability over inputs. It forms one of the three major categories of machine learning, together with supervised learning and reinforcement. Semisupervised learning, a similar type, makes use of methods that are supervised and unsupervised.

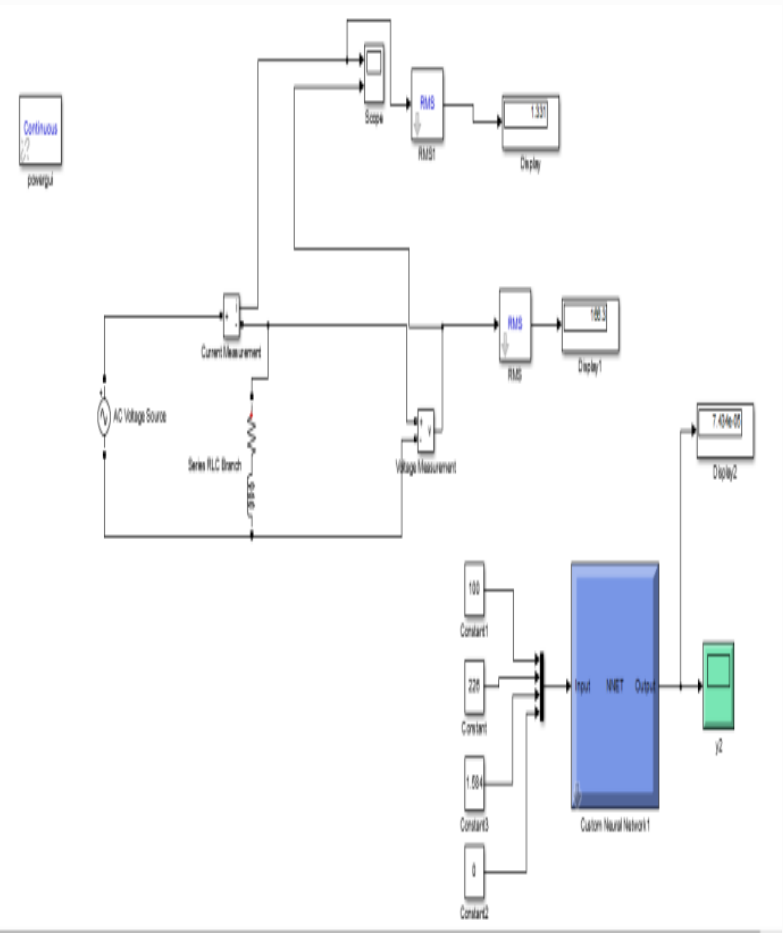

Volume 02 Issue 06 June 2020

Fig 4. Model for normal values

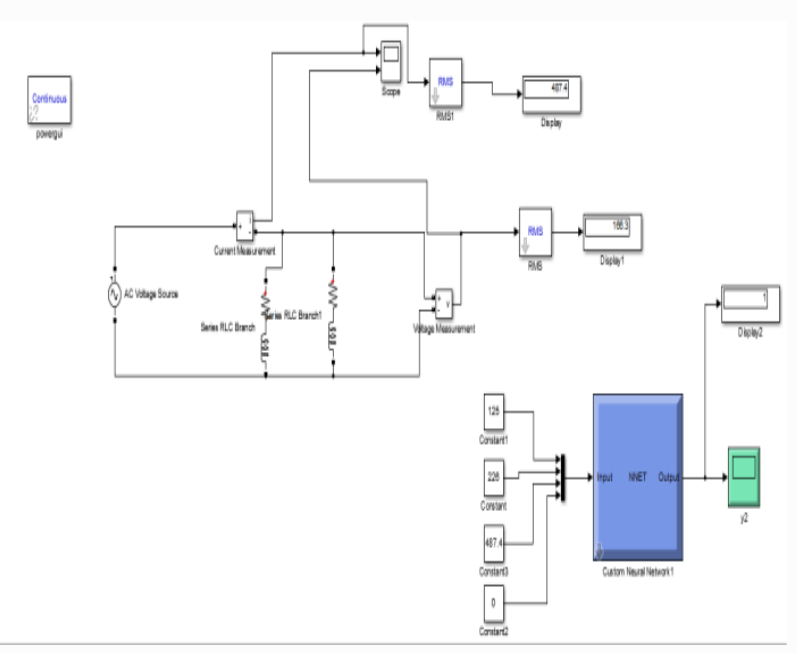

Fig.5.Model for abnormal values

The simulation model above is used to obtain sample data. We can find the load current values by adjusting the source voltage and the load resistance. The data's were trained in Artificial Neural Network to observe performances here.

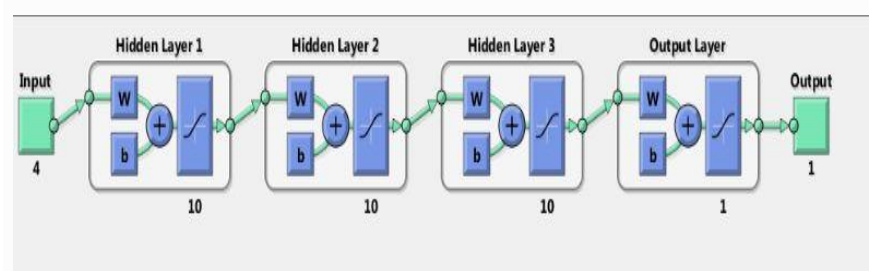

Fig.6.Neural Network Architecture

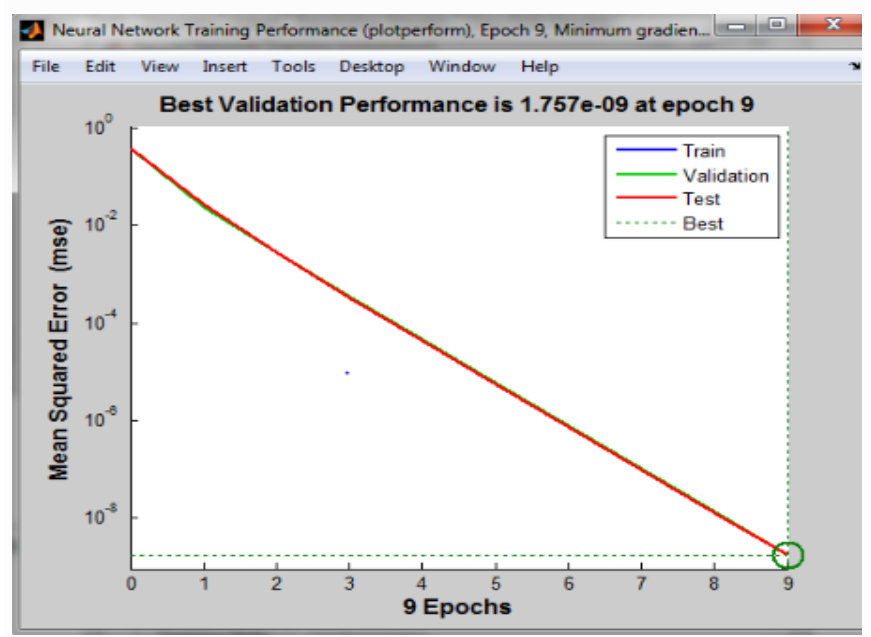

Fig.7.Performance of neural network 
www.rspsciencehub.com

This trained data's is implemented in Numerical Relay Development Environment Board.

\section{NRDE BOARD}

Numeric relay development Environment Board (NRDE) consisting of newly built relay and computer-driven test bench system based on hardware in the loop. The hardware is designed to accept analog signal from LAB VIEW's DAQ card. Software package provided and injected into the relay using the NI 9263 converter modules from National Instrument and the software package from Lab VIEW. The performance of the relay scheme is analyzed in the computer by the capability of relay event capture and serial communication with the computer. Differential scheme for percentage bias generator is implemented and tested in setup. Results show the hardware efficiency is reliable and accurate.

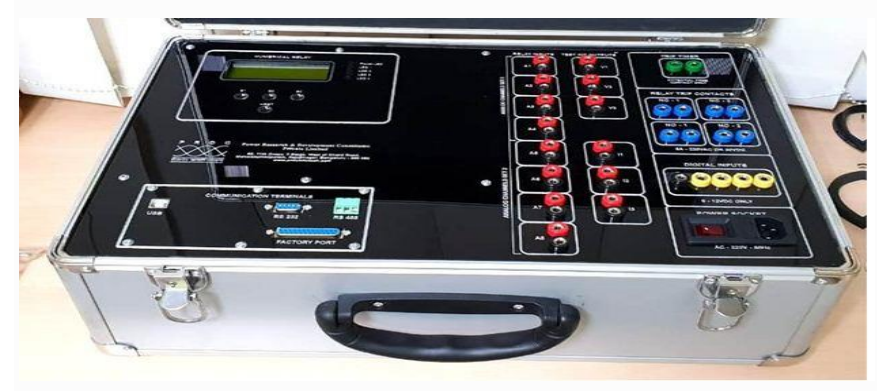

Fig.8.NRDE Development board

The developed code is downloaded in NRDE Software(pc loader application).

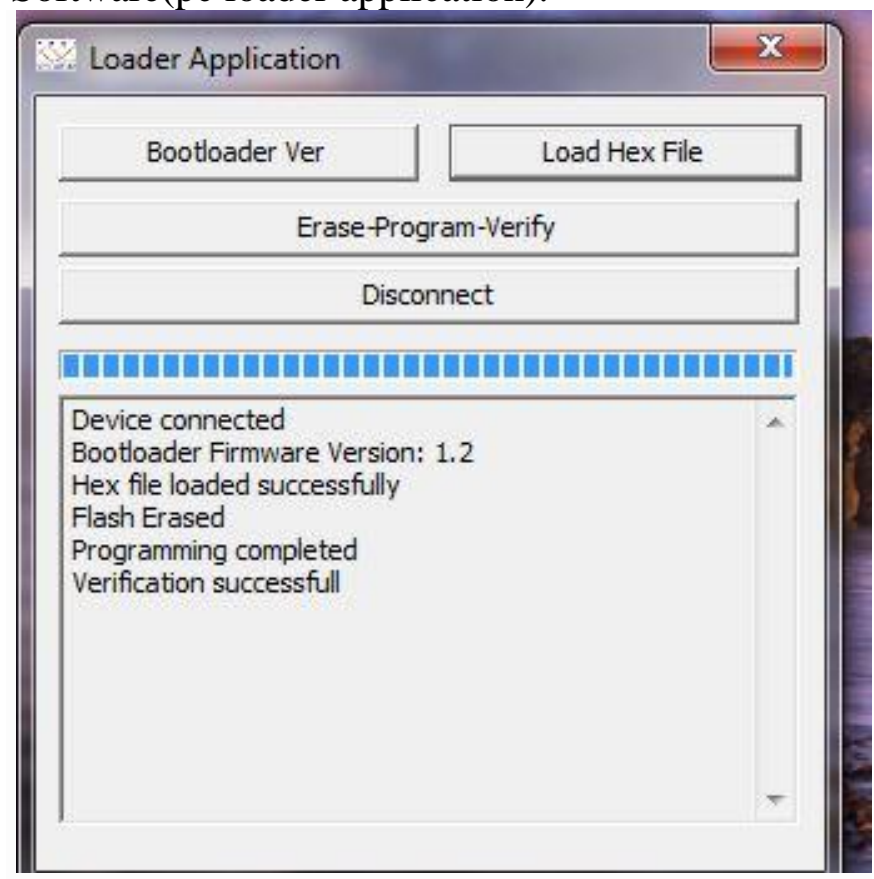

Fig.9.loader application
Volume 02 Issue 06 June 2020

After completion of loader process the trip command was observed in NRDE hardware kit.

\subsection{STEADY STATE ANALYSIS}

Voltage Amplitude can be entered in Va, $\mathrm{Vb}$, and Vc for ADC channels, similarly Current Amplitude values may be entered in Ia, Ib, Ic. Harmonics order and percentage of Harmonics distortion can be entered in the section Harmonics. Provision for entering Fundamental Frequency (F), Sample No./Cycle (N), how many cycles to display $(\mathrm{Cg})$, Sampling Rate (Fs).

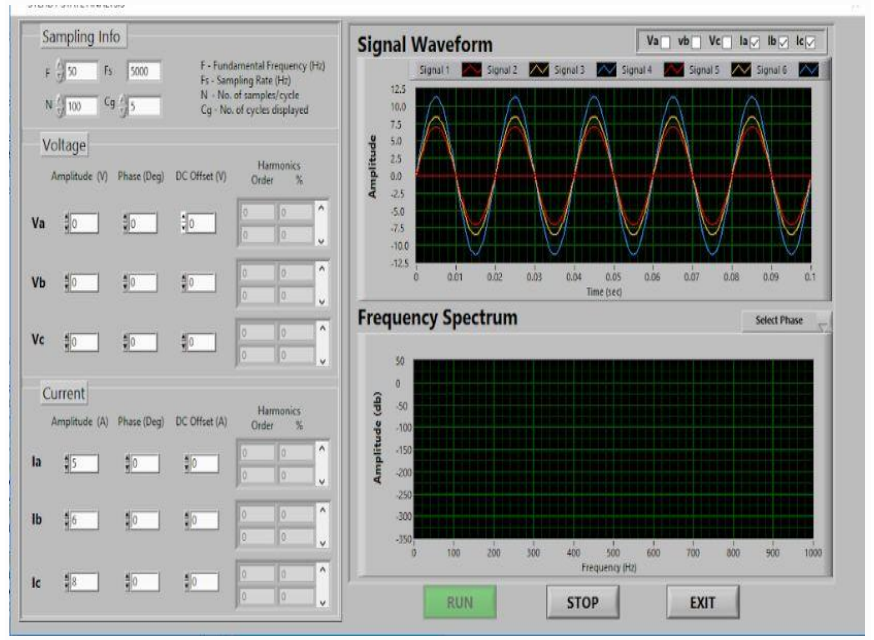

Fig.10.waveforms of over current

If DFT sampling algorithm records are collected in the NRDE application, the user may use NRDE Event Downloader Application software to store sampling data in the user computer using the following steps.

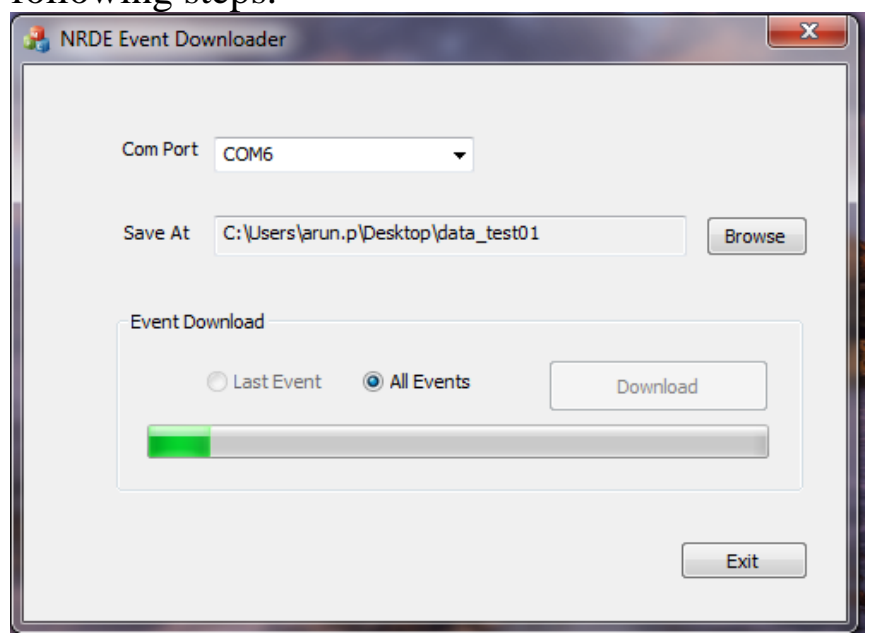

Fig.11.Sampling records 


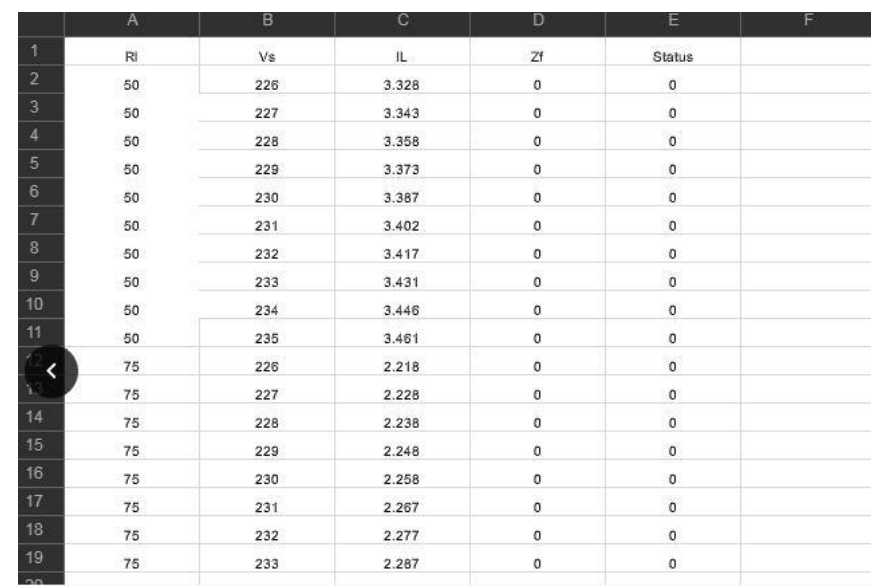

Fig.12.sampling records in excel sheet

\section{RESULT}

The performance of overcurrent relay is achieved by NRDE hardware kit.The voltage and current channels are connected.

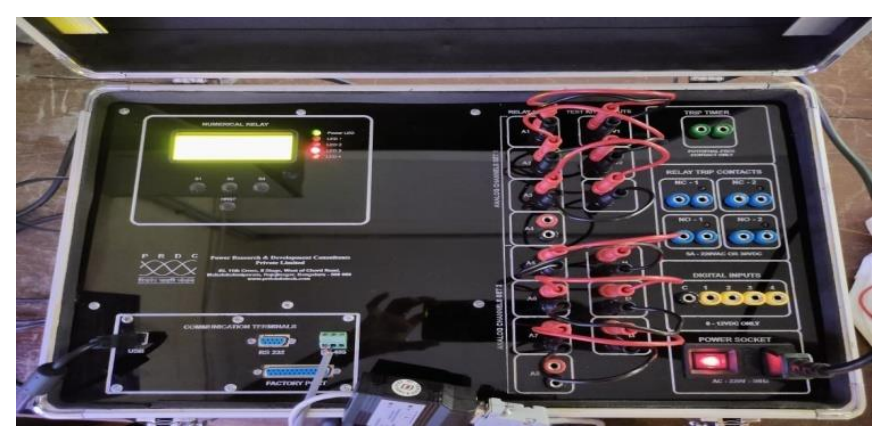

Fig.13.NRDE Board connection

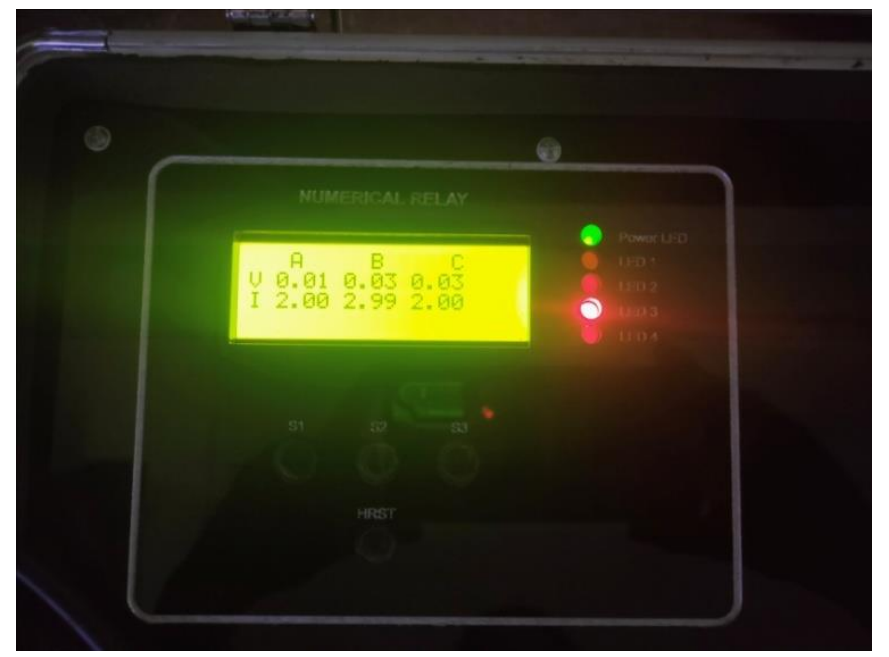

Fig.14.Tripped signal
This paper uses NRDE Board to presents numerical overcurrent relay security. The MPLAB $X$ IDE v2.05 interface encodes an over-current relay system. The secondary of $\mathrm{CT}$ is attached to the NRDE board and processed by running the over-current program. In the NRDE Test software a trip command was observed when over-current condition was reached.

\section{Conclusion}

Using MPLAB platform, an overcurrent relay program is encoded. With the aid of MATLAB Simulation, supercurrent is achieved. By varying the source voltage and load resistance, we can find the normal load current values (In rms) and when the short circuit element is inserted into that model, the load circuit value is automatically increased and treated as overcurrent. Overcurrent data is trained in Artificial Intelligence(Neural Network) and implemented through coding in the NRDE Board. In NRDE testing software, a trip command is observed when overcurrent condition is reached. The social effect of this initiative is an unexpected power loss which can be prevented due to fault. For existing power grids, stable and protected power network can be achieved.

\section{References}

[1] PROF. D.P. Kothari Application of Neural Networks to power system centre for energy studies lit. delhi. new delhi-110016, india.

[2] D.P. Sengupta et al.,"Recent Advances in

Control and Management of Energy Systems", Ch. 15 by P.K. Dash, Interhey 1993.

[3]M.El-Sharkawi and D. Niebur(Eds.),'TEm Tutorial Come on ANNs with Applications to Power Systems", \% TP 112-0.624

[4] L. Srivastava, S.N. Slngh and J. Sharma,"ANN Aapphation in Power systems: An overview and Key Issues", Proc. Int. \& d . CERA 97, pp. 39743.

[5] M. Qunanovic, et al.,"Unsupervised/supervised Learning 24. Concept for 24 Hour Load Forecasting", IEE Proc. Pt. C, Vol.14, No.4, July 1993, p . 311-318.

[6] M. Singh, B. K. Panigrahi and R. Mukherjee, "Optimum Coordination of overcurrent relays 
using CMA-ES algorithm," in Proc. 2012 IEEE International Conference on Power Electronics, Drives and Energy Systems (PEDES), pp. 1-6.

[7] D. K. Singh and S. Gupta, "Optimal coordination of directional overcurrent relays: A genetic algorithm approach," in Proc. 2012 IEEE Students' Conference on Electrical, Electronics and Computer Science (SCEECS), pp. 1-4.

[8] IEEE Guide for the Application of Current Transformers Used for Protective Relaying Purpose'. IEEE Std. C37, 2007, 16, p. 110

[9] M. Y. Shih, C. A. C. Salazar, and A. C. Enrquez, "Adaptive directional overcurrent relay coordination using ant colony optimisation," IET Gener. Transm. Distrib., vol. 9, no. 14, pp. 20402049, 2015.

[10] M. H. Hussain, S. R. R. Rahim, and I. Musirin, "Optimal overcurrent relay coordination: a review," Procedia Engineering, vol. 53, pp. 332336, 2013. 